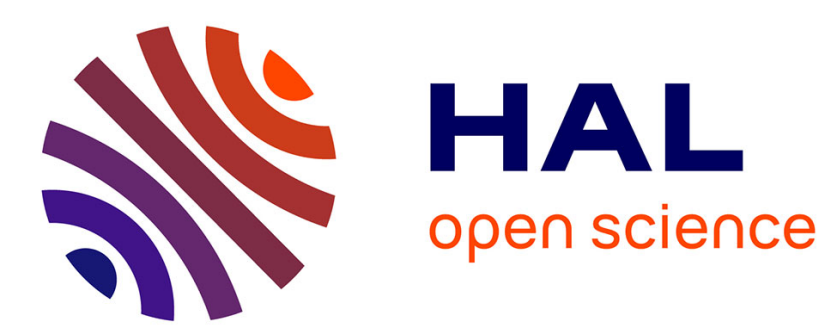

\title{
Compressive Sensing-Based Metrology for Micropositioning Stages Characterization
}

Ning Tan, Cédric Clevy, Guillaume Laurent, Nicolas Chaillet

\section{To cite this version:}

Ning Tan, Cédric Clevy, Guillaume Laurent, Nicolas Chaillet. Compressive Sensing-Based Metrology for Micropositioning Stages Characterization. IEEE Robotics and Automation Letters, 2016, 1 (2), pp.638 - 645. hal-02868179

\section{HAL Id: hal-02868179 https://hal.science/hal-02868179}

Submitted on 15 Jun 2020

HAL is a multi-disciplinary open access archive for the deposit and dissemination of scientific research documents, whether they are published or not. The documents may come from teaching and research institutions in France or abroad, or from public or private research centers.
L'archive ouverte pluridisciplinaire HAL, est destinée au dépôt et à la diffusion de documents scientifiques de niveau recherche, publiés ou non, émanant des établissements d'enseignement et de recherche français ou étrangers, des laboratoires publics ou privés. 


\title{
Compressive Sensing-Based Metrology for Micropositioning Stages Characterization
}

\author{
Ning Tan ${ }^{1}$, Cédric Clévy ${ }^{1}$, Guillaume J. Laurent ${ }^{1}$ and Nicolas Chaillet ${ }^{1}$
}

\begin{abstract}
High accuracy is a necessary condition for reliable performance of MicroPositioning Stages (MPSs). However there are various sources of errors that affect their precision. Characterization is a prior step to calibration for compensating systematic errors so as to improve the positioning accuracy. In this letter, the Compressive Sensing (CS) theory is applied to characterize system errors of MPSs. This method could be flexibly collaborated with any sensors and applicable to widespread micro-systems where the motions and errors are required to be measured. CS (1) improves the data acquisition and processing in terms of time, and (2) could be employed as an interpolating strategy to efficiently replace the lookup tables. As a case study, the CS-based method is applied to characterize the position-dependent errors of an $X Y$ serial MPS. Experimental results show that the method is able to retrieve the microscale positions with largely shortened time and high precision. The spent time for data acquisition and processing is shortened by more than $84 \%$ for $X$ stage and $82 \%$ for $Y$ stage. These results are especially promising for microscale purposes where the system behavior is varying and difficult to characterize.
\end{abstract}

Index Terms-Micro/Nano Robots, Calibration and Identification, Automation at Micro-Nano Scales, Computer Vision for Other Robotic Applications

\section{INTRODUCTION}

$\mathbf{S}$ CALING down to microworld has brought many benefits to technology development. Meanwhile, difficulties are emerging due to the specificities at such a small scale. For example, high operation accuracy is demanded in a variety of microtasks [1], such as, microassembly [2], biological micromanipulation [3], micromachining [4], etc. Considering many factors, such as success rate, speed, and contamination, these tasks usually rely on MicroPositioning Stages (MPSs) with automatic control instead of manual operation [5]. Microtask platforms usually consist of one or several MPSs. The types, structures, and numbers of the MPSs depend on the tasks to be fulfilled.

Unfortunately, the inherent imperfections in off-the-shelf MPSs could be of the noticeable issues for achieving micrometer accuracies. Some manufacturers provide statistical

Manuscript received: August, 31, 2015; Revised November, 23, 2015; Accepted January, 26, 2016.

This paper was recommended for publication by Editor Yu Sun upon evaluation of the Associate Editor and Reviewers comments. This work was funded by the Franche-Comté region, OSEO and partially supported by the Labex ACTION project (contract ANR-11-LABX-0001-01) and by the French RENATECH network and its FEMTO-ST technological facility.

${ }^{1}$ All authors are with FEMTO-ST Institute, UFC-ENSMM-UTBMCNRS, Université de Franche-Comté, 25000 Besançon, France tanningrobotics@gmail.com, cclevylfemto-st.fr, guillaume. laurent@ens $2 \mathrm{~m}$. fr,

nicolas.chailletefemto-st.fr

Digital Object Identifier (DOI): see top of this page. specifications, for example the positioning repeatability and sensor resolution. However, these data are not sufficient to ensure a good accuracy of a micro end-effector attached to the MPS because some of imperfections are position-dependent. For instance, for a $20 \mathrm{~mm}$ long tip mounted on such a stage, the positioning error could reach $3 \mu \mathrm{m}$ at the end-point in the perpendicular direction to the motion owing to the yaw deviation. In addition, MPSs usually have limited Degrees-ofFreedom (DoF). The assembly of several of them is required to meet specific needs. Commonly, grippers or probes are also fastened onto the stages as end-effector. These assemblies of tools introduce some geometric errors further. For example, if the perpendicularity error between two $X$ and $Y$ axes is $0.1^{\circ}$, a $1 \mathrm{~cm}$ motion along $Y$ could result in a $17 \mu \mathrm{m}$ error along $X$ which is significant at the microscale. To achieve a favourable accuracy, assembly and position-dependent errors must be measured, quantified, and compensated. However, the error characterization of the MPS requires a stepsize down to a few micrometers or even nanometers. Therefore, to characterize the stroke of a MPS in centimeter range, a great amount of points need to be measured and processed, which is a fairly timeconsuming procedure. Moreover, during long measurements, the system's behavior is subjected to environmental perturbations, which induces a mix between the intrinsic performance of the system itself and external influences. Thus it is very difficult to really understand the intrinsic behavior because accurate measurements usually take a long time. Our previous works [6], [7] enabled to understand better the behavior of micro and nano positionning robots and to improve a lot their performance through robot calibration approach. They also shown that measurement is the most critical issues where the measurement duration is an important technical trade-off. The long-term measurements (several hours are required to have good enough data) are detrimental not only to usability, but also to the performances themselves, which are brittle to more influential effects and increase the risk of coupling effects acting on the robot accuracy. Hence, it is of great importance to reduce the measurement duration as well as keeping the high quality of data. To shorten the implementing (measuring + processing) time for measurements, in this letter, compressive sensing is applied to characterize geometric errors along the axes. The CS-based method can be flexibly collaborated with any sensors and applicable to widespread mechatronic systems where the motion and errors are need to be measured. To showcase the method, an $X Y$ MPS formed by two micropositioning stages is chosen as the case study because such kind of structure is very popular in microscale applications. In short, the two main contributions are: 
- The CS technique is applied to characterization of microscale motions and errors.

- The proposed CS-based framework is implemented to a typical study case of serial MPSs, which contributes to understand their behaviors.

The remainder of this letter is organized as follows. Section II introduces the basic principle of CS and some related works. Section III discusses the working mechanism of the proposed characterization framework. The experimental setup and concerned physical quantity of the case study are presented in Section [V] Section V presents the characterization results and corresponding discussions. Finally, the letter is concluded in Section VI

\section{COMPRESSIVE SENSING AND RELATED WORK}

\section{A. Compressive sensing}

Compressive sensing is a breakthrough signal processing technique enabling to acquire and to recover a finite signal from a set of random measurements, instead of high-density measurements limited by the Nyquist rate, to carry out highly accurate metrology [8], [9], [10]. This theory of sampling is based on the fact that realworld signals typically have a sparse representation in a certain transformed domain, which means most physical phenomena are compressible in some transform basis, e.g. Fourier Transform (FT), Discrete Cosine Transform (DCT), wavelets, etc.

Given an unknown signal $f=\left[f_{1}, \ldots, f_{n}\right]^{T} \in \Re^{n \times 1}$, it is assumed that it can be represented as a linear combination of certain basis functions by depicted as:

$$
f=\Psi \theta,
$$

where $\Psi=\left[\psi_{1}, \ldots \psi_{n}\right] \in \Re^{n \times n}$ is basis matrix and $\theta$ is corresponding vector of coefficients. The basis functions must be suited to a particular application. Most of the coefficients $\theta$ are assumed to be effectively zeros (i.e., sparse) and have $s$ nonzero coefficients (i.e., $s$-sparse).

To recover the signal $f$, it takes $m$ times linear measurements to sample the original signal, which can be depicted by the following formula:

$$
h=\Phi f,
$$

where $\Phi=\left[\phi_{1}, \ldots, \phi_{m}\right]^{T} \in \Re^{m \times n}$ is the measurement matrix and $h \in \Re^{m \times 1}$ is the measurement result vector. If $m=n$, the original signal $f$ could be reconstructed by directly solving linear equations. CS concerns $m \ll n$ which means to recover the original signal using much fewer measurements.

Combining with Eq. 11. Eq. 2 can be rewritten as

$$
h=\Phi \Psi \theta=A \theta,
$$

where $A=\Phi \Psi$. Eq. (3) is obviously an under-determined equation. Normally it is impossible to find the unique solution for this equation. However, if the $\Phi$ and $\Psi$ are properly designed and the original signal is sparse as well, $f$ could be successfully recovered if $A$ satisfies the Restricted Isometry Property (RIP) [8] which is a sufficient condition for sparse solution. Usually, the RIP of a given matrix is difficult to check, whereas it has been verified that random matrices satisfy the RIP with high probability. Alternatively, the sparse solution can also be ensured in terms of incoherence between $\Phi$ and $\Psi$ which is defined as

$$
\mu(\Phi, \Psi)=\sqrt{n} \cdot \max _{1 \leq i, j \leq n}\left|\left\langle\phi_{i}, \psi_{j}\right\rangle\right|,
$$

To ensure the sparse solution, $\mu(\Phi, \Psi)$ should be as small as possible. It is known that random matrices are largely incoherent with any fixed basis. And, spikes and sinusoids are maximally incoherent [8]. Hence, in this paper we choose $\Phi$ as a matrix of random binary (i.e. impulses) and $\Psi$ as a compression basis of sinusoids.

Then the sparse representation $\hat{\theta}$ can be obtained via the $l_{1}$ norm minimization readily by solving a convex optimization problem through linear programming:

$$
\hat{\theta}=\arg \min \|\hat{\theta}\|_{l_{1}} \quad \text { s.t. } \quad A \hat{\theta}=h,
$$

where

$$
\|\hat{\theta}\|_{l_{1}}=\sum_{i=1}^{n}\left|\hat{\theta}_{i}\right| .
$$

Having the coefficients $\hat{\theta}$, the signal $f$ can be recovered by computing the following formula:

$$
g=\Psi \hat{\theta}=\sum_{i=1}^{n} \psi_{i} \hat{\theta}_{i}
$$

where $\hat{\theta}=\left\{\hat{\theta}_{i}\right\}_{i=1}^{n}$ and $g$ is the reconstructed version of $f$.

\section{B. Literature review}

Thanks to its merits and universality, the CS theory has been applied to a variety of areas, especially to communication [11], computational biology [12], medical imaging [13], remote sensing [14], astronomy [15], and the like.

In addition, CS has been extended to some fields a little bit far from conventional application areas. For example, CS was applied to surface characterization and metrology [16]. In [17], authors addressed post-silicon characterization of the unique gate delays and their timing distributions on each manufactured IC by CS.

The CS technique has been applied to the micro-nano area. In [18], a system including a micro-mirror array acquired only a fraction of the samples by projecting subsets of image pixels onto a prism. Unfortunately, the sampling was not ideal for CS due to practical limitations, and the sensed signal did not necessarily meet the strict sparsity demands. A compressive feedbacks based non-vector space control approach was proposed for improving the accuracy of AFM based nanomanipulations. Instead of sensors, the local image was used as the feedback to a nonvector space controller to generate a closed-loop control for manipulation [19].

The CS theory has also been introduced to robotics where most of them are correlated to environmental mapping where the robots acted as the mobile agents to collect local information [20], [21]. For example, the mission design strategies for mobile robots whose task was to perform spatial sampling of a static environmental field were considered in the framework of CS [22]. Besides, an unpublished work [23] presented 
a lightweight method for spatial sampling of environmental phenomenon using a large group of robots. Data were gathered using simple random walks and the field was reconstructed using CS. In [24], the CS technique was applied to make robot know its position and construct the environment map with minimal sensing information. A person detection and tracking method was proposed for a mobile robot by fusing the data from Radio Frequency Identification (RFID) and stereo camera which was used for person detection based on the CS theory. Less Haar-like features were extracted from the compressive domain to represent the person by a sparse measurement matrix [25].

To the best of our knowledge, CS has not been applied for the positioning errors metrology and characterization in real robotic systems, especially in MPSs.

\section{CS-BASED GEOMETRIC ERRORS CHARACTERIZATION}

As mentioned in Section $\mathbb{I}$ in the cases of requiring large amount data, the data processing time is really a tough problem, and this is always the case in micro-nano areas. Because the characterization of errors of the MPS required the stepsize down to a few micrometers or even nanometers, to characterize the stroke of the MPS in centimeter range, a great amount of points need to be measured and processed, which is a fairly time-consuming procedure.

Inspired by the essential merit of compressive sensing introduced in Section II], a novel characterization method is proposed through combining the position sensor and CS theory. The architecture of the framework is illustrated in Fig. 1 and the general procedure is as follows:

- Step 1: To recover the error function $f \in \Re^{n \times 1}$, a measurement matrix $\Phi \in \Re^{m \times n}$ is designed to sample $m$ random positions. The measurement matrix should be not only random but also realistic and practical. Since the error function here is a kind of 1D spacial signal, the most convenient way is to randomly measure a few positions. The positions of measurements are determined by generating a random permutation. The chosen points of the random positions are set as "1" and stored in $\Phi$. The rest points are set as " 0 " meaning that the coordinates of such points won't be taken. Since all the chosen points are on the straight line, the characterization trajectories are designed to easily follow these points without the need of particular trajectory planning. The random positions are sorted and stored in sequence. For example, suppose the $m$ measured positions are at $2 \mathrm{nd}$, 5 th, $\ldots,(n-1)$ th instants, the measurement matrix is

$$
\Phi=\underbrace{\left[\begin{array}{cccccccc}
0 & 1 & 0 & 0 & 0 & \cdots & 0 & 0 \\
0 & 0 & 0 & 0 & 1 & \cdots & 0 & 0 \\
& & \ddots & & & \vdots & & \\
0 & 0 & 0 & 0 & 0 & \cdots & 1 & 0
\end{array}\right]}_{n}\} m .
$$

Such a randomly generated binary matrix is incoherent to the DCT transform matrix. That is to say, there is a lack of correlation between the sensing modality embodied by the rows of $\Phi$ and the basis formed by the columns of $\Psi$ [8], [9], [26].

- Step 2: The MPS is controlled to move to the random positions given prior. Meanwhile, the corresponding positions are measured by the external position sensor and stored in the memory for the follow-up use. It is worth noting that the position sensor here is not of a specific kind but could be any ones adequate for the applications.

- Step 3: After finishing the data acquisition and processing, the coordinates of random positions, $p_{m}=$ $\left[p_{m, 2}, p_{m, 5}, \ldots, p_{m, n-1}\right]$, can be retrieved.

- Step 4: Then the corresponding errors $h$ at the random positions can be obtained through calculating $h=p_{t}-p_{m}$ where $p_{t}=\left[p_{t, 2}, p_{t, 5}, \ldots, p_{t, n-1}\right]$ is the target coordinates.

- Step 5: The defined $\Psi$, the designed $\Phi$, and the measured $h$ are proceeded to solve the $l_{1}$-norm minimization problem in (5), and then the sparse transform $\hat{\theta}$ is obtained. The matrix $A$ becomes

$$
A=\left[\begin{array}{cccc}
\Psi_{2,1} & \Psi_{2,2} & \cdots & \Psi_{2, n} \\
\Psi_{5,1} & \Psi_{5,2} & \cdots & \Psi_{5, n} \\
\vdots & \vdots & \vdots & \vdots \\
\Psi_{n-1,1} & \Psi_{n-1,2} & \cdots & \Psi_{n-1, n}
\end{array}\right] .
$$

- Step 6: The total errors of the axis are recovered through calculating $g=\Psi \hat{\theta}$ as the reconstruction of $f$.

- Step 7: Go back to Step 1 and repeat the process until finishing the characterization of all the axes.

It is worth noting that the position sensor could be whatever fitting for specific applications. The $\Psi$ should be also congruent with the physical quantity to be measured. For finite signals, the FT and DCT are two commonly used transforms. The FT implicitly assumes a periodic extension of the signal, which results in discontinuity at the boundary [27]. The DCT assumes an antisymmetric extension of the signal, which results in continuous boundaries. Hence, the DCT is chosen in our case study.

Moreover, the framework could mantle a wide range of applications. For example, the framework could be extended to measure and characterize other physical quantities. For that, the corresponding sensors, measurement matrices, and sparse transforms should be adapted to the specific applications.

\section{CASE STUdy}

The position-dependent errors along axes are significant characteristics of precise positioning stages. These errors are due to the geometric nature of the axes. For macroscale robotics, this type of errors is usually neglected in calibration which mainly focuses on kinematic parameters identification or elastic deformation. However, these errors become significant at the microscale, especially in Cartesian MPSs.

The mobile stages of many MPSs are guided based on friction principles. Their positioning performances depend on the qualities of fabrication, plays, weight of the axes and so on. The stages are usually equipped with internal sensors and are individually closed-loop controlled in actuation layer. But depending of the location of the sensors in the actuation chain, 


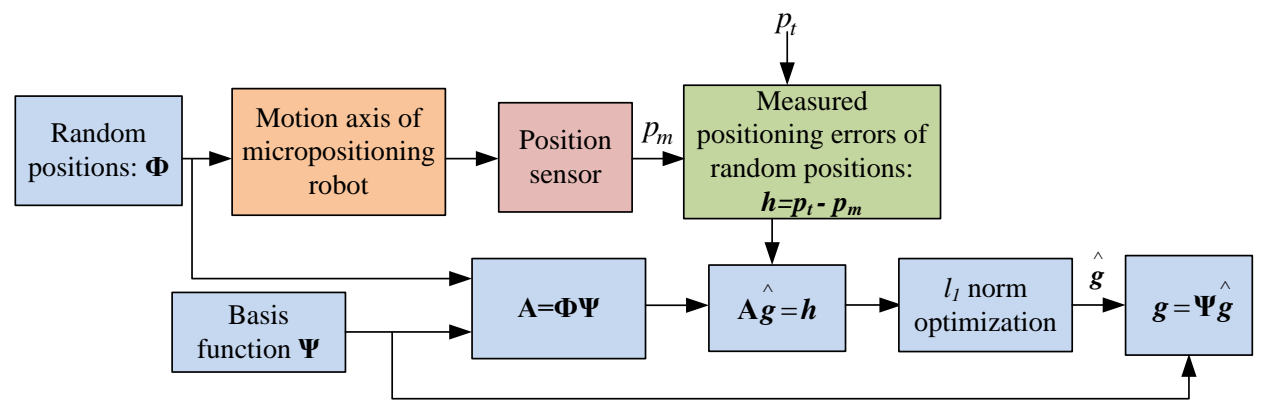

Fig. 1. Architecture of the MPS error characterization based on CS.

the feedback control can not reject some sources of errors. Moreover, stages assembly errors can not be compensated using only proprioceptive sensors.

\section{A. Experimental setup}

In this letter, an $X Y$ serial MPS is chosen as a case study of the proposed characterization method because this kind of structure is representative for many systems commonly used in performing micromanipulation. The pictures of the whole experimental setup is shown in Fig. 2(a) where the system is mounted on a anti-vibration table. The two translation stages are PI M-111.1DG equipped with MercuryTM C-863 controllers. Table I gives the specifications of the translation stages $X Y$ according to the datasheets.

The external measuring system for characterization consists of a $1024 \times 768$ video camera (AVT STINGRAY F-125C), a microscope lens (Optem zoom 70XL), an objective with $10 \times$ magnification and a Pseudo-Periodic encoded Pattern (PPP) (Fig. 2(b)). The upper goniometer (M-GON40-U) and lower goniometer (M-GON40-L) are used for adjusting the parallelism between the pattern and the camera.

TABLE I

SPECIFICATIONS OF XY TRANSLATION STAGES IN DATASHEET

\begin{tabular}{lc}
\hline Stage & PI M-111.1DG \\
\hline Travel range & $15 \mathrm{~mm}$ \\
Resolution & $50 \mathrm{~nm}$ \\
Unidirectional repeatability & $100 \mathrm{~nm}$ \\
Pitch angle deviation & $\pm 150 \mu \mathrm{rad}$ \\
Yaw angle deviation & $\pm 150 \mu \mathrm{rad}$ \\
Backlash & $2 \mu \mathrm{m}$ \\
Thread pitch & $0.4 \mathrm{~mm}$ \\
Driving mechanism & Leadscrew \\
\hline
\end{tabular}

\section{B. External measuring system}

The PPP algorithm [28] is suitable for microscale measurement because its high resolution and long ranges in the two directions of the image plane. Hence, it is chosen with the vision system as the sensing tool for microscale position measurement in the case study. The PPP algorithm is based on an encryption of a binary code over a PPP. The position is retrieved by combining fine and coarse measurements that are complementary. First, the coding allows absolute but coarse coordinate transformations of the image reference frame into actual positions on the observed part of the pattern. Image processing returns the line and column orders necessary for the fine position through phase computations. Second, the PPP allows a high level of interpolation through phase measurements that lead to subpixel resolution. This process gives the position with typical resolution of $10^{-3}$ pixels and an indeterminacy equal to the wavelength of the pattern.

As the period of the pattern is precisely known $(4 \mu \mathrm{m}$ in the present case), the measurement is intrinsically selfcalibrated. Then there is no need to calibrate the imaging system. In the present case, the sensing range of the measuring system is limited by the size of the pattern that is $9.5 \mathrm{~mm}$ in $x$-axis and $4.2 \mathrm{~mm}$ in $y$-axis. The reproducibility of the visual measurement has been experimentally evaluated and is better than $10 \mathrm{~nm}$. More details about the algorithm and the fabrication of the pattern can be found in [29].

\section{Position-dependent errors}

According to the specification, the driving mechanism of the stage is leadscrew. So the errors along the axis could be foreseen somehow based on the mechanical properties. The error curves are functions of axis coordinates, and the functions are different from one axis to another, so measurements of these errors for every axis are necessary. As depicted in Fig. 3 each stage is controlled to reach appointed target coordinates $\left\{p_{x t}, p_{y t}\right\}$. The camera captures the images of the pattern

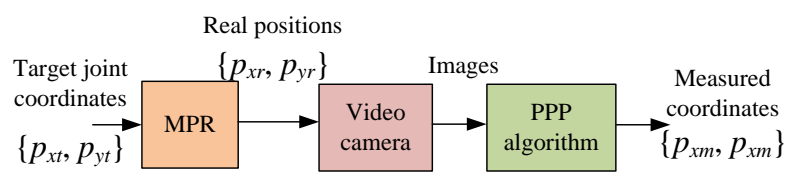

Fig. 3. Block diagram of position-dependent errors characterization.

in the real positions $\left\{p_{x r}, p_{y r}\right\}$. The images are processed subsequently with the PPP algorithm so as to obtain measured coordinates $\left\{p_{x m}, p_{y m}\right\}$. The position-dependent errors are calculated by comparing the measured positions (estimation of real positions) with the targeted positions (positions to be reached). The error at a given point contains two parts: the first part induced by $X$ stage's motion and the second part by $Y$ stage's motion. To decouple the two parts, the trajectories for error characterization are designed as 1-DoF straight lines, that is, one stage is moving, while another stage is kept static. The error components are defined as $e f_{x i}$ and $e f_{y i}$ when 


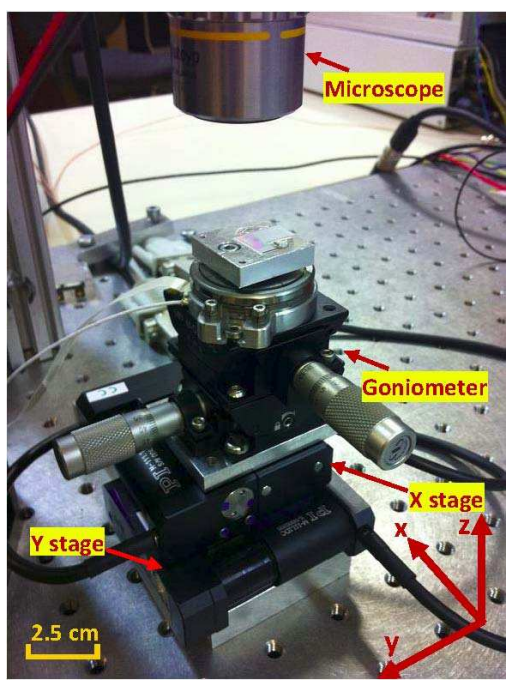

(a) Global view

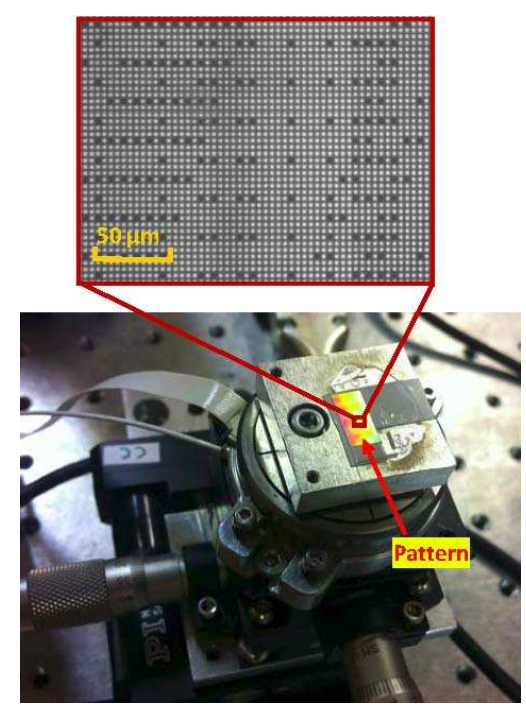

(b) Local view

Fig. 2. Experimental setup of the $X Y$ serial MPS for case study.

moving forward in $i$ direction and are defined as $e b_{x i}$ and $e b_{y i}$ when moving backward. As long as these components are characterized, the error at any point in the workspace is known [28].

\section{EXPERIMENTAL VALIDATION AND DISCUSSIONS}

\section{A. Full data characterization}

Firstly the full data of position-dependent errors are measured by using the PPP method. Fig. 4 shows the differences between $p_{x t}$ and $p_{x m}$, namely the errors of $e f_{x x}$. It can be seen that errors vary cyclically. Such behavior is reasonably assumed due to systematic turn-to-turn nature inherent in the leadscrew. The thread pitch of the stage is $400 \mu \mathrm{m}$, so the cyclical error repeats with the same period. It can also be observed that the periods of the errors in forward and backward motions of the $X$ stage are the same, but the magnitudes are slightly different. The driving system does not work symmetrically and makes a systematic error between forward and backward motions that corresponds to the backlash of $2 \mu m$ as specified by the manufacturer. Moreover, positiondependent errors appear not only in the driving direction but also in the lateral one. Fig. 5 shows the coupling errors in $y$ direction when only $X$ stage is moving forwards and backwards. It is seen that the coupling errors have the same period as the errors of driving direction. In the strokes of 9500 and $4200 \mu \mathrm{m}$, the position measurements are taken with step size of $5 \mu \mathrm{m}$, and a total of 3801 data from $X$ stage and 1681 data from $Y$ stage are obtained for every cycle including a forward and a backward motion.

\section{B. Characterization based on CS}

The following results are on the outcomes of applying the CS-based framework. The corresponding signal $f$ is the position-dependent errors to be characterized. The 300 random measurements used for recovering the original $f$ are showed

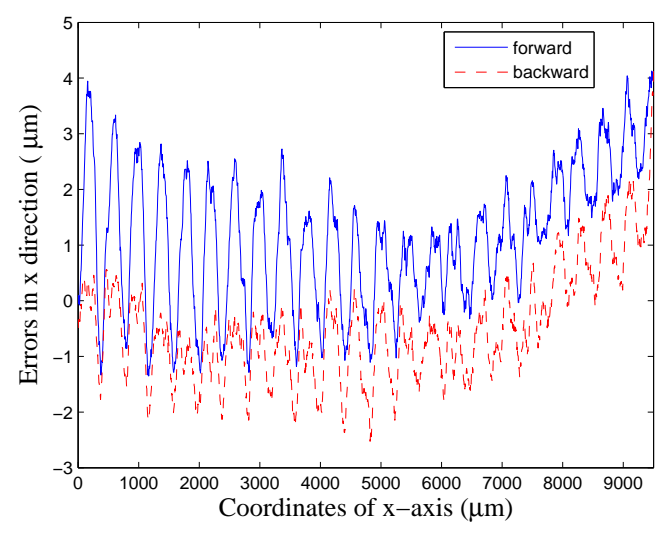

Fig. 4. Full data of errors $e f_{x x}$ (or $p_{x t}-p_{x m}$ ) in $x$ direction when $X$ stage is moving forwards and backwards in one cycle.

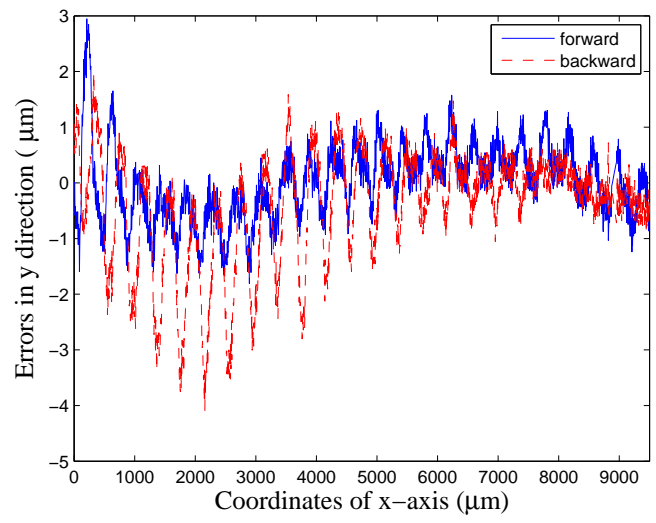

Fig. 5. Full data of coupling errors $e f_{x y}$ (or $\left.0-p_{y m}\right)$ in $y$ direction when $X$ stage is moving forwards and backwards in one cycle.

with the original in Fig.6(a). Fig. 6(b) shows the comparison of original and recovered position-dependent errors of $\mathrm{x}$-axis, from which the two curves can be seen quite close (i.e., 
overlapped). This result preliminarily proves that the CS-based framework is able to recover the errors efficiently with very few measurements (i.e., 300) compared to the total number of original data (i.e., 1901).

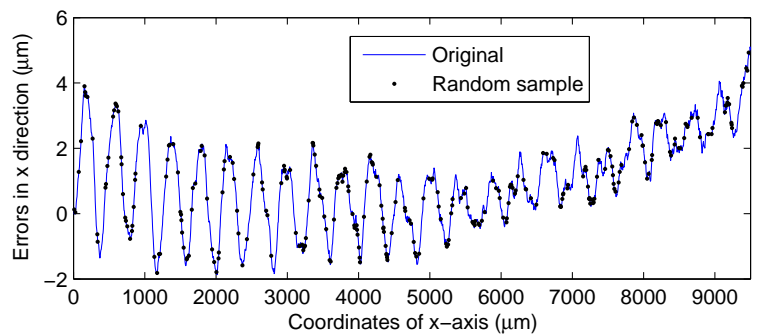

(a)

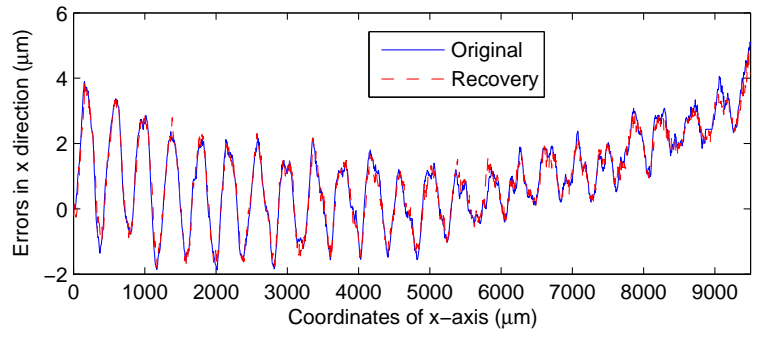

(b)

Fig. 6. Errors $e f_{x x}$ (i.e., $f$ or $p_{x t}-p_{x m}$ ) in $x$ direction with 300 random measurements when $X$ stage is moving forwards. (a) Random samples (i.e., $y$ ) and the original (i.e., $f$ ); (b) Recovered errors (i.e., $g$ ) and the original.

Fig. 7 gives the DCT transform coefficients of the original errors in normal order and random order as well for enhanced visibility. It is showed that most of the dots are (or nearly) zeros which means the original errors are sparse in this domain. The differences between the original errors and the
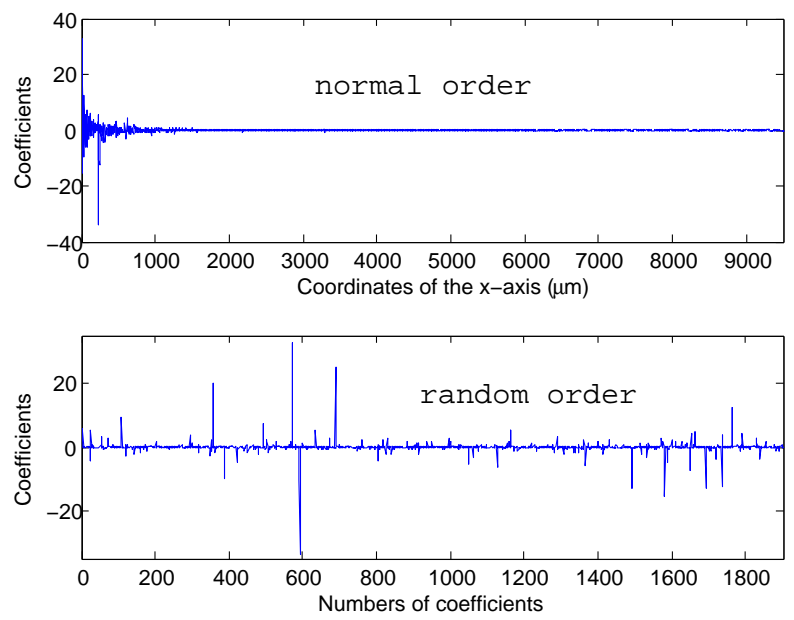

Fig. 7. Sparse coefficient vector $\hat{\theta}$ of errors in $x$ direction when $X$ stage is moving forwards (arranged in normal and random orders for enhanced visibility).

recovered ones are shown in Fig. 8 in terms of four sets of random measurements, namely 100, 300, 600, and 900. Using measurements of 100 random points, the recovery errors are about $-2 \sim 3 \mu \mathrm{m}$ which are not satisfactory at this small scale. Increasing the number to 300 gives a much better recovery
TABLE II

MEAN ABSOLUTE VALUES, MAXIMUM ABSOLUTE VALUES AND STANDARD DEVIATIONS OF THE RECOVERY ERRORS $(g-f)$ IN $x$ DIRECTION WHEN $X$ STAGE IS MOVING FORWARDS

\begin{tabular}{ccccc}
\hline Percentage of measurements $(\%)$ & 5.26 & 15.78 & 31.56 & 47.34 \\
\hline Mean absolute error $(\mu \mathrm{m})$ & 0.512 & 0.198 & 0.092 & 0.047 \\
Maximum absolute error $(\mu \mathrm{m})$ & 3.088 & 1.186 & 0.650 & 0.560 \\
Standard deviation $(\mu \mathrm{m})$ & 0.655 & 0.270 & 0.141 & 0.085 \\
\hline
\end{tabular}

with errors of $\pm 1 \mu \mathrm{m}$. By doubling (to 600) and tripling (to 900) the number of measurements, the improved performances of recovery are able to be achieved further. As observed in the figure, the improved errors are within $\pm 0.25 \mu \mathrm{m}$ which are small enough for many applications. All these numbers are less than half data (i.e., 1901) which means that the measurement burden can be slashed markedly. From the zoom region, it can be observed that the recovery of the unmeasured errors is quite good rather than using the interpolation techniques and the lookup table in conventional handling. Table II provides the corresponding mean absolute values, maximum absolute values and standard deviations of the recovery errors. It is observed that the three sets of values decrease a lot where for example, standard deviation decreases from $0.65 \mu \mathrm{m}$ to $0.25 \mu \mathrm{m}$ when the number of measurements is tripled. With less than half data, the standard deviation declines to small than $0.1 \mu \mathrm{m}$. Besides, the coupling errors in $y$ direction
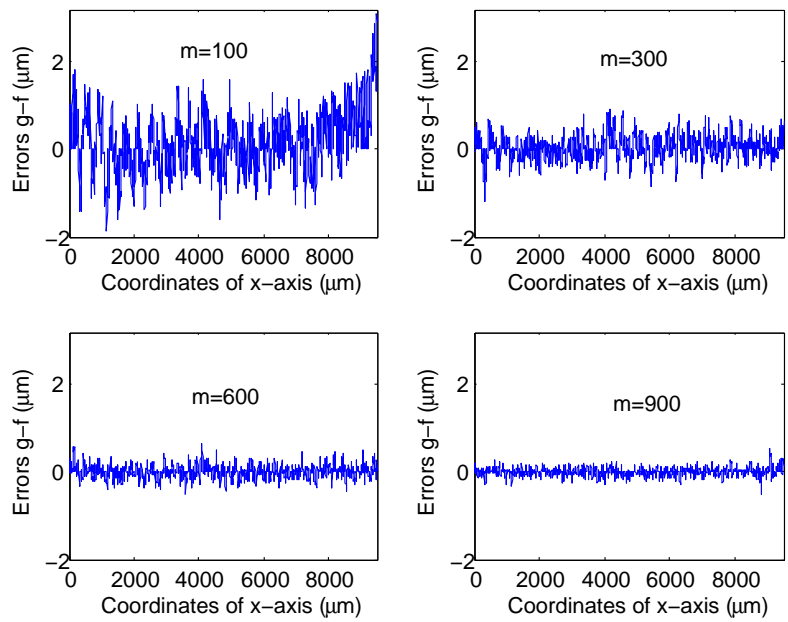

Fig. 8. Recovery precisions $(g-f)$ in $x$ direction when $X$ stage is moving forwards.

can also be reconstructed efficiently as demonstrated in Fig. 9 The CS-based method not only guarantees high quality of reconstruction of the position-dependent errors, but also reduce the time consumption efficiently. Table III presents the comparisons of time consumption between the full scan and the CS-based method in data acquisition and processing processes. The 600 and 300 random measurements in one cycle are used for recovery of errors along $X$ and $Y$ stages, respectively. Thanks to the CS-based method, the spent time of data acquisition and processing is shortened by $84.23 \%$ for $X$ stage and $84.18 \%$ for $Y$ stage. So the original time (more than 11.5 hours) decreases to less than 2 hours. Since the number 
of the full data in $Y$ stage is less than half of data in $X$ stage, half random measurements are acquired to reconstruct the position-dependent errors. In this case, more than 5 hours spent fall sharply to less than 1 hour.

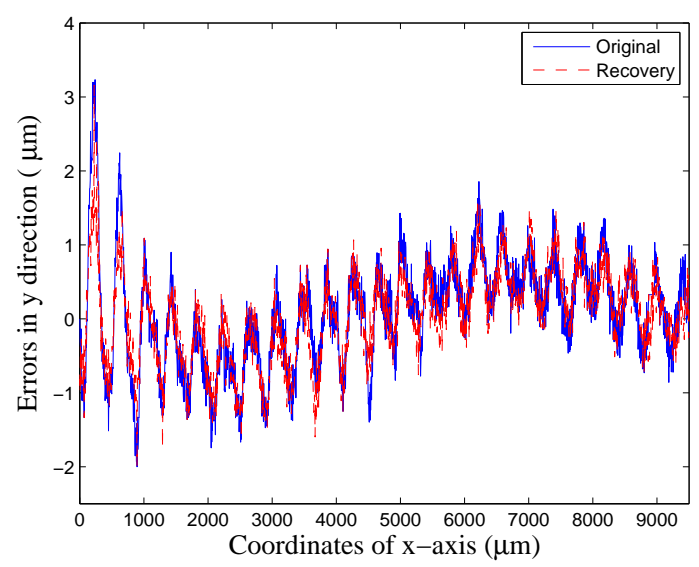

Fig. 9. Recovery of coupling errors $e f_{x y}$ (or $0-p_{y m}$ ) with 300 random measurements in $y$ direction when $X$ stage is moving forwards.

\section{CONCLUSiOnS}

In this letter, a novel characterization framework based on CS was proposed. This methodology has several advantages: 1) The CS-based method decreases the required measurements in data acquisition process, thereby shortens the time needed for data processing. 2) Because the CS can retrieve positioning errors at the positions not measured, this framework could be employed as an interpolating strategy to efficiently replace the lookup table conventionally used. 3) The framework is flexible to collaborate with any position sensors. 4) The framework is extendable to measure and characterize other physical quantities at the micro-nano scale in collaboration with other kinds of sensors.

As a showcase, the position-dependent errors in the $X Y$ serial MPS were characterized by using the proposed framework. Experimental results illustrated that the recovered positiondependent errors were fit to the original ones with fitting errors within $\pm 1 \mu \mathrm{m}$ or even $\pm 0.25 \mu \mathrm{m}$ by increasing the number of measurements. These results verified the efficiency of the proposed method in terms of precision and interpolating capability. Furthermore, the time consumed in data acquisition and processing processes was enormously cut down by nearly $84 \%$ for $X$ stage and $82 \%$ for $Y$ stage, which verified the efficiency of the proposed method in terms of speed. The future work would be the calibration of MPSs based on the characterized errors.

\section{ACKNOWLEDGMENT}

We are very grateful to Stéphane Chrétien for its helpful discussions and suggestions. We wish to thank both the reviewers and the editors for providing us valuable feedback on this document.

\section{REFERENCES}

[1] Y. Maeda and M. Iwasaki, "Mode switching feedback compensation considering rolling friction characteristics for fast and precise positioning," IEEE Trans. Ind. Electron., vol. 61, pp. 1123-1132, Feb. 2014.

[2] E. Avci, K. Ohara, C.-N. Nguyen, C. Theeravithayangkura, M. Kojima, T. Tanikawa, Y. Mae, and T. Arai, "High-speed automated manipulation of microobjects using a two-fingered microhand," IEEE Trans. Ind. Electron., Aug. 2014.

[3] Y. Zhang, K. K. Tan, and S. Huang, "Vision-servo system for automated cell injection," IEEE Trans. Ind. Electron., vol. 56, pp. 231-238, Jan. 2009.

[4] A. Tüysüz, C. Zwyssig, and J. W. Kolar, "A novel motor topology for high-speed micro-machining applications," IEEE Trans. Ind. Electron., vol. 61, pp. 2960-2968, Jun. 2014.

[5] N. Chaillet and S. Régnier, Microrobotics for Micromanipulation. WileyISTE, 2010.

[6] N. Tan, C. Clévy, G. J. Laurent, and N. Chaillet, "Accuracy quantification and improvement of serial micropositioning robots for in-plane motions," IEEE Transactions on Robotics, vol. 31, no. 6, pp. 497-1507, 2015.

[7] N. Tan, C. Clévy, and N. Chaillet, "Calibration of nanopositioning stages," Micromachines, vol. 6, no. 12, pp. 1856-1875, 2015.

[8] E. Candès and M. Wakin, "An introduction to compressive sampling," IEEE Signal Processing Mag., vol. 25, no. 2, pp. 1053-5888, 2008.

[9] R. G. Baraniuk, "Compressive sensing," IEEE Signal Processing Mag., vol. 24, pp. 118-121, Jul. 2007.

[10] S. Chrétien, "An alternating 11 approach to the compressed sensing problem," IEEE Signal Processing Letters, vol. 17, no. 2, pp. 181-184, 2010.

[11] G. Gui, W. Peng, and F. Adachi, "High-resolution compressive channel estimation for broadband wireless communication systems," Int. J. Commun. Syst., 2012.

[12] M. Mohtashemi, H. Smith, F. Sutton, D. Walburger, and J. Diggans, "Sparse sensing DNA microarray-based biosensor: Is it feasible?," in IEEE Sensors Applications Symp., (Limerick, Ireland), pp. 127-130, 2010.

[13] J. P. Haldar, D. Hernando, and Z.-P. Liang, "Compressed-sensing MRI with random encoding," IEEE Trans. Med. Imag., vol. 30, pp. 893-903, Apr. 2011.

[14] J. Ma, "Single-pixel remote sensing," IEEE Geosci. Remote. S., vol. 6, pp. 199-203, Apr. 2009.

[15] Y. Wiaux, L. Jacques, G. Puy, A. Scaife, and P. Vandergheynst, "Compressed sensing imaging techniques for radio interferometry," Astrophysics, vol. 395, pp. 1-10, Feb. 2009.

[16] J. Ma, "Compressed sensing for surface characterization and metrology," IEEE Trans. Instrum. Meas., vol. 59, pp. 1600-1615, Jun. 2010.

[17] F. Koushanfar, P. Boufounos, and D. Shamsi, "Post-silicon timing characterization by compressed sensing," in IEEE/ACM Int. Conf. ComputerAided Design, (San Jose, CA, USA), pp. 185-189, 2008.

[18] Y. Pfeffer and M. Zibulevsky, "A micro-mirror array based system for compressive sensing of hyperspectral data," tech. rep., Technion-Israel Institute of Technology, 2010. CS-2010-01.

[19] B. Song, J. Zhao, N. Xi, H. Chen, K. W. C. Lai, R. Yang, , and L. Chen, "Compressive feedback-based motion control for nanomanipulationtheory and applications," IEEE Trans. Robot, vol. 30, pp. 103-114, Feb. 2014.

[20] S. Huang and J. Tan, "Compressive mobile sensing in robotic mapping," in IEEE/RSJ Int. Conf. Intel. Robot. Syst., (St. Louis, MO, USA), pp. 3070-3075, 2009.

[21] S. Huang and J. Tan, "Adaptive sampling using mobile robotic sensors," in IEEE/RSJ Int. Conf. Intel. Robot. Syst., (San Francisco, CA, USA), pp. 1668-1673, 2011.

[22] R. Hummel, S. Poduri, F. Hover, U. Mitra, and G. Sukhatme, "Mission design for compressive sensing with mobile robots," in IEEE Int. Conf. Robot. Autom., (Shanghai, China), pp. 2362-2367, 2011.

[23] S. Poduri, G. Marcotte, and G. S. Sukhatme, "Compressive sensing based lightweight sampling for large robot groups." http://www.researchgate.net/publication/228440936_Compressive_ sensing_based_lightweight_sampling_for_large_robot_groups.

[24] S.-Y. Fu, X.-K. Kuai, R. Zheng, G.-S. Yang, and Z.-G. Hou, "Compressive sensing approach based mapping and localization for mobile robot in an indoor wireless sensor network," in IEEE Int. Conf. Network. Sensing. Control, (Chicago, IL, USA), pp. 122-127, 2010.

[25] L. Wang, S. Jia, X. Li, and S. Wang, "RFID and vision based person tracking of a mobile robot using improved compressive tracking," in 
TABLE III

COMPARISONS OF TIME CONSUMPTION BETWEEN FULL SCAN AND THE CS-BASED METHOD FOR ERRORS CHARACTERIZATION OF X AND Y MICROPOSITIONING STAGES IN ONE CYCLE

\begin{tabular}{cccc}
\hline Action & Time for full scan (hours) & Time for CS-based method (hours) & Improvement rate $(\%)$ \\
\hline X stage: Data acquisition & 3.17 (3801 meas.) & $0.50(600$ meas.) & 84.23 \\
X stage: Data processing & 8.47 (3801 meas.) & $1.34(600$ meas.) & 84.18 \\
Y stage: Data acquisition & 1.40 (1681 meas.) & $0.25(300$ meas.) & 82.14 \\
Y stage: Data processing & 3.74 (1681 meas.) & $0.67(300$ meas.) & 82.09 \\
\hline
\end{tabular}

IEEE Int. Conf. Inform. Autom., (Yinchuan, China), pp. 1171-1176, 2013.

[26] K. Bryan and T. Leise, "Making do with less: an introduction to compressed sensing," SIAM Review, vol. 55, no. 3, pp. 547-566, 2013.

[27] R. Rubinstein, A. M. Bruckstein, and M. Elad, "Dictionaries for sparse representation modeling," Proceedings of the IEEE, 2010.

[28] N. Tan, C. Clévy, G. J. Laurent, P. Sandoz, and N. Chaillet, "Characterization and compensation of $\mathrm{XY}$ micropositioning robots using vision and pseudo-periodic encoded patterns," in IEEE Int. Conf. Robot. Autom., (Hong Kong, China), pp. 2819-2824, 2014.

[29] V. Guelpa, G. J. Laurent, P. Sandoz, J. G. Zea, and C. Clévy, "Subpixelic measurement of large 1D displacements: Principle, processing algorithms, performances and software," Sensors, vol. 14, pp. 50565073, Mar. 2014. 